http://dx.doi.org/10.12795/PH.1990.v05.i01.17

\title{
Las representaciones gráficas: medio para visualizar la estructura de la frase
}

\author{
Ángela Gracia Menéndez
}

El objeto de la presente disquisición es la representación gráfica de la estructura sintáctica de la frase. Las primeras representaciones, aplicadas por los filólogos alemanes Gustav Billroth y Franz Kern, datan del siglo XIX. Coseriu (1980: 49, 56)1.

Es evidente que las representaciones gráficas o esquemas contribuyen eminentemente a visualizar las estructuras sintácticas de la lengua como sistema. También parece obvio que se debe sobre todo a la posibilidad de servirse de dos dimensiones lo que facilita tener en cuenta la estructura jerárquica de la sintaxis ${ }^{2}$. Debido a la complejidad del tema nos limitaremos a algunos ejemplos que pueden considerarse significativos para la evolución de la teoría gramatical.

Tesnière denominó la representación bidimensional del conjunto de las relaciones sintácticas existentes en una oración «estema» y lo definió como:

«la représentation graphique de l'arquitecture des connexions», Tesnière, Esquisse d'une Syntaxe Structurale, (19824:3).

En el siglo XIX tuvo inicialmente un aplicación pedagógica, que fue adquiriendo paulatinamente una finalidad científica, hasta llegar a ser una herramienta indispen-

\footnotetext{
${ }^{1}$ Dentro del estructuralismo americano, Coseriu menciona la aplicación de «árboles» por Eugene Nida, Morphology. The Discriptive Analysis of Words, Ann Arbor, 1946; Ch. C. Fries, The Structure of English, New York, 1952, H.A. Gleason, An Introduction to Descriptive Linguistics, New York, 1958 y Ch. F. Hockett, A Course in Modern Linguistics, New York, 1958. Coseriu, Un précurseur méconnu de la syntaxe structurale (1980:48).

${ }^{2}$ Un ejemplo de un formalismo unidimensional es el enfoque del estructuralista danés Otto Jespersen. El logró señalar la jerarquía estructural por medio de números. Philosophy of Grammar, London, 19639, obra que se publicó en 1924, y posteriormente Analytic Syntax, New York 1969', publicado en 1937. Heringer, Theorie der deutschen Syntax, (19732:15-19).
} 
sable para visualizar no sólo la estructura sintáctica de una frase sino incluso poner de relieve toda la teoría gramatical implícita.

La novedad de los estemas utilizados por Tesnière radica en su concepción de predicado ${ }^{3}$ que marca, sin duda, un hito en la historia de la teoría gramatical. La asunción de un núcleo verbal ${ }^{4}$ dio lugar al desarrollo de la gramática de valencias que motivó una serie de posteriores planteamientos y abrió nuevas perspectivas dentro de la disciplina linguiística.

Como demuestra Coseriu ya anteriormente a Tesnière algunos gramáticos recurrieron a la aplicaciòn de esquemas como medio para visualizar la estructura de una frase $\mathrm{e}^{5}$. Sin embargo, al continuar con la concepción de predicado aristotélica no lograron una aproximación adecuada para el estudio de la lengua.

La diferencia de los estemas utilizados por Tesnière estriba, sin embargo, en que él no mantiene la separación tradicional de la frase en sujeto y predicado por considerarlo erróneo desde un punto de vista gramatical. (1982:15) Tesnière rechaza todo tipo de razonamiento que provenga de la lógica en contra de la concepción de un nudo verbal como núcleo de la frase, aduciendo que la lógica no tiene nada que ver en cuestiones linguiísticas y la califica de:

«survivance non encore éléminée, de l'époque, que va d'Aristote à Port Royale, ou toute la Grammaire était fondée sur la logique», (1982:103).

Su rechazo de un enfoque lógico renovará esencialmente la teoría gramatical en el siglo XX, ya que permite equiparar el sujeto a los demás complementos del predicado de la frase, poniendo fin a una tradición que colocaba el sujeto en una función privilegiada en relación con los demás complementos, tradición que hoy día -desafortunadamente- no está todavía completamente erradicada, como lo demuestra la Gramática Generativa.

Con la concepción de un nudo verbal Tesnière logra superar categorías lógicas dentro de la linguística, así como venían reivindicando algunos gramáticos del siglo XIX, adaptando la metodología de la teoría gramatical a las exigencias de la lengua.

Desde los «Eléments de Syntaxe structurale» en 1959 la teoría gramatical experimenta un desarrollo, que pese a la escasa repercusión que tuvo en sus primeros años tras su aparición -comparándola con «Syntactic Structures» o "Aspects of the Theory of Syntax»- comienza a dar fruto sólo unas dos décadas después y sobre todo

${ }^{3}$ Tesnière, L., Eléments de Syntase Structurale, (1982:104).

4 Tesnière, L., Eléments de Syntaxe Structurale, (1982:14-15).

${ }^{5}$ Coseriu, E., Un précurseur méconnu de la syntaxe structurale, (1980:49, 50, 56, 60). 
en un círculo de lingüistas alemanes, a quienes les sirvió de base para numerosos trabajos, en los que continuaron la tarea iniciada por Tesnière ${ }^{6}$.

Consistía, pues, en un primer paso, en determinar la valencia para cada verbo, especificando el número de lugares vacíos. Y posteriormente enumerar el tipo de complementos (caso, grupo preposicional o frase completiva con dass, $o b$, etc.), lo que resultó ser mucho más difícil de lo que parecía al principio. Destacamos tan sólo el diccionario de Engel/Schumacher, «Kleines Valenzlexikon deutscher Verben», Tübingen 1978, así como el diccionario bilingüe francés-alemán de Busse/Dubost, Französisches Verblexikon, Stuttgart, 19832.

Pero la mayor contribución para la linguística se aportó con la discusión que surgió a raíz de cómo había de ser concebido el término «valencia» y que cuestionaba un planteamiento de carácter fundamental para el problema:

Si el concepto de la valencia había de ser tratado dentro de la sintaxis, éste se convertiría en una categoría formal. Sin embargo, si debería ser estudiado dentro de la semántica, la valencia constituiría una categoría ontológica. Eroms (1981:8). Se configuraron estas dos posturas esbozadas a lo largo de la discusión, de las que la segunda aportó aspectos fundamentales para el desarrollo de la lingüística al introducir conceptos semánticos en cuestiones consideradas puramente estructurales, es decir, sintácticas y por consiguiente, descuidadas hasta entonces.

En efecto, Tesnière nunca se cuestionó tales problemas, aunque -dado su interés por asuntos meramente estructurales- cabe suponer que Tesnière hubiera optado por una aplicación estrictamente formal ${ }^{7}$.

Su planteamiento, sin embargo, ha supuesto aproximaciones que permitieron llegar a establecer al menos una correlación -aunque poco precisa- entre sintaxis y semántica, sin poder determinar la prioridad de una u otra ${ }^{8}$.

En el siguiente apartado pasamos en primer lugar la gramático Johann Friedrich Billroth (1808-1836), que fue uno de los primeros que utilizó esquemas en su Lateinische Syntax für die obern Klassen gelehrter Schulen, Leipzig 1832, con el objetivo de facilitar a los alumnos de latín la pertenencia de los adjetivos a determinadas partes de la oración. Billroth desarrolló en su sintaxis una teoría de las determinaciones gramaticales, introduciendo una terminología que más tarde fue criticada por Franz Kern,

\footnotetext{
${ }^{6}$ La teoría de valencias fue prácticamente ignorada por completo en los EE.UU, en donde la lingüística se limitaba al perfeccionamiento de la Gramática Generativa y a las pocas contestaciones de aquellos que cuestionaban la renuncia a la semántica. Tan sólo Charles J. Fillmore pone en duda la separación de sujeto y predicado dentro de su gramática de caso que constituye una crítica a la teoría standard de Chomsky. Pese a remitirse a Tesnière sus casos semánticos en una estructura profunda poco tienen que ver con la teoría de valencias. La categoría sintáctica «caso», dentro de una estructura superficial, es sustituida por relaciones semánticas profundas, como «agente» etc.

Charles J. Fillmore, Plädoyer für Kasus, (1977²:24), en: Kasus-theorie, Werner Abraham, ed. Wiesbaden, Athenaion.

7 Tesnière no hace en ningún momento referencia a cuestiones semánticas, relacionadas con el fenómeno de la valencia verbal.

${ }^{8}$ Peter Eisenberg es uno de los pocos lingüistas que ven la valencia de un verbo es determinada por el significado, es decir, con los objetos que están relacionados por el significado del predicado de una frase. Grundriss der Deutschen Grammatik, (1986:82-83), Stuttgart.
} 
como veremos más adelante, pero que -afortunadamente-no fue adoptada por los demás gramáticos de su época, según Coseriu (1980:56).

Billroth utilizó el término de «frase vestida» (bekleideter Satz) con el que hacía referencia a la frase completa con todos los objetos. A ese término le oponía el de «frase desnuda» (nackter Satz) con el que denominaba el sujeto de la frase y el predicado (Prädikatsverb), adoptando pues, la concepción de predicado tradiconal.

Así establece que la «frase desnuda», cuyo esquema vemos en la página 264 (1), es Miltiades reddidit. Miltiades tiene función de sujeto, y reddidit es el predicado de la frase.

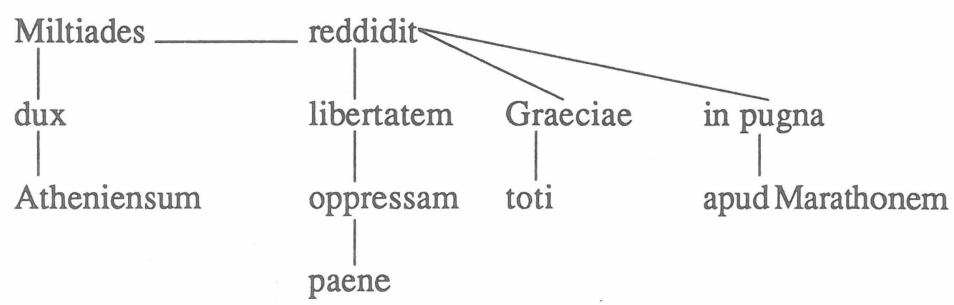

En el análisis, Billroth mantiene la separación tradicional de la frase en sujeto y predicado. Esta concepción se refleja en el esquema (1) en que el sujeto Miltiades está a un mismo nivel junto al predicado de la frase reddidit, mientras que los objetos parten del predicado a un nivel inferior, ya que éstos se consideran más próximos al predicado. Billroth hace una distinción entre sujeto y objetos, suprimida en la teoría de valencia, pero sin embargo, no diferencia la relación sintáctica de régimen, como es el caso entre reddidit y el objeto en acusativo libertatem oppressam paene, de la relación atributiva (y por consiguiente no de régimen) entre Miltiades y dux Atheniensum. En este caso es evidente que no se puede equiparar la función completiva del objeto a la atributiva de la aposición dux Atheniensum, ya que ésta puede perfectamente omitirse, sin que la frase resulte incorrecta.

Pese a las objeciones que hacemos a los esquemas de Billroth, coincidiendo éstas con los argumentos aportados por Tesnière, hemos querido esbozar la concepción de predicado por considerarla característica para la época -teniendo en cuenta que la fecha de aparición es el año 1832. Debido a la bisección de la frase en sujeto y predicado, el esquema de Billroth es un ejemplo de la concepción de predicado tradicional.

Por otro lado nos permitirá apreciar mejor lo innovador de los esquemas utilizados cinco décadas después por Franz Kern, que a juzgar por las referencias a su terminología, tenía que conocer la obra de Billroth.

La primera obra de Franz Kern (1830-1894) Die Deutsche Satzlehre, Berlín 1883, no contenía aún representaciones gráficas, no obstante, ha de interesarnos su concepción de predicado. Franz Kern recurre a la imagen de un árbol9":

${ }^{9}$ Kern compara también la frase a la construcción de una casa:

«Der viele reiche Bestimmungen aller Art in sich enthaltene Satz (Hauptsatz) ist zu vergleichen mit dem Bau eines Hauses; die in ihm enthaltenen Begriffe und sprachilchen Verdindungen (auch Nenbensätze) 
«Aber viel lieber vergleiche ich den lebendigen Organismus der menschliche Rede des Satzes mit einem Baum, der zwei deutlich geschiedene Aeste, die sich nachher auf das mannigfachste gliedern, aus sich hervortreibt». Kern, Die deutsche Satzleh$r e,(1883: 26)$.

Kern rechaza por motivos estéticos la terminología utilizada por Billroth, como ya mencionábamos, y sostiene que ni es adecuado ni de buen gusto imaginarse los complementos de una frase como camisas, falda o chaleco. (1883:96).

En su intento de explicar la metáfoca utilizada de «árbol», Kern atribuye una función clave al predicado de la frase.

«Das finite Verbum nämlich wäre gleich dem noch ungeteilten Stamm, die beiden Aeste den Bestimmungen der Verbalperson (dem Subjektswort) und den Bestimmungen des Verbalinhaltes (den Prädikatsbestimmungen)» (1883:26).

Deducimos de la imagen del árbol una serie de ideas: el verbo finito que tiene la función de predicado equivale al tronco del árbol. Es del tronco del que parten las ramas: el sujeto y los objetos. A pesar de que Kern aún distingue entre sujeto y demás complementos, -los objetos-, el sujeto ya no está al mismo nivel que el predicado (comparándolo con Billroth), sino que sujeto y objetos parten de aquel en un segundo plano.

Kern reconoce el papel importante del predicado y sostiene que no es comparable a las demás partes de la oración, sino que el predicado constituye la oración por sí mismo en su forma más simple.

«So ist das finite Verbum gar kein Satzteil in dem Sinne, wie es Objekt oder Subjektswort sind, es ist selber schon der Satz in seiner einfachsten Form, dem alles Übrige als Bestimmung sich anschliesst». Kern, Die Deutsche Satzlehre, (1883:65).

Kern ilustra la función del predicado, aduciendo una cita de Humboldt y que comenta de la siguiente manera:

«Humboldt sagt (S. 371) mit vollem Recht vom Verbum, dass es sowohl durch das Subjekt als durch das Objekt, in deren Mitte es stehe, in seinem Begriffe vervollStändigt werde». Kern, Die Deutsche Satzlehre, (1883:65).

Kern denomina el predicado «Träger des Satzes» (portador de la frase) o «Ausdruck des Zustandes» (expresión de un estado) y añade que en las lenguas flexivas siempre tiene que aparecer, (1883:66). El calificativo de «Grundlage des

wären aber gleich den schon fertig gemachten Teilen den behauenen und geformten Steinen, den Türen und Fenstem, die nun hier eingefügt werden, aber auch für andere ähnliche Gebäude verwendbar gewesen wären; und es heisst wohl die Vergleichung nicht zu Tode hetzen, wenn man die Präpositionen und Konjunktionen vergliche mit den Năgeln und Klammern, die in jedem Bauwerk zur Verwendugn kommen.

Kern, Die Deutsche Satzlehre, (1883:25). 
ganzen Satzgebäudes» (1883:66), es decir, base de la construcción de la frase, que atribuye al predicado, demuestra el papel fundamental que desempeña para Kern en la formación de la frase.

Lo que sí interesa destacar es que no considera el predicado «parte de la oración» como lo son el sujeto y los objetos, lo que implicaría ser complemento de otro elemento, sino que es un elemento que no es exigido por ningún otro elemento de la frase. En ese sentido su enfoque es significativo y hace que su concepción de predicado sea en cierto modo comparable al nudo verbal de Tesnière.

La importancia fundamental del predicado de la frase se refleja también en los esquemas, según Coseriu (1980:60), que utiliza en su obra Grundriss der Deutschen Satzlehre, Berlín 1884. Kern recurre con un objetivo pedagógico a una representación gráfica en Zur Methodik des deutschenu nterrichts, Berlín, 1883, por primera vez y posteriormente en el Grundriss (1884), que denomina «Satzbild», imagen de la frase, $\mathrm{y}$ «anschauliche Darstellung».

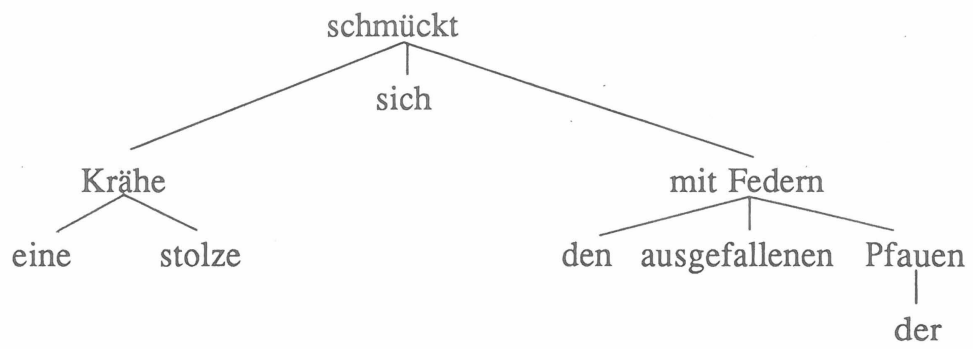

Por lo que a la imagen del árbol se refiere, se trata en realidad de un árbol invertido, pero con analogías obvias al estema de Tesnière:

El núcleo de la oración es el predicado schmückt sich, que en este caso es un verbo reflexivo.

De él parten dos ramificaciones, el primero Krähe y el segundo mit Federn, y a su vez de Krähe otras dos ramificaciones que son el artículo, en este caso indeterminado, y el adjetivo, con función de modificar el sustantivo. Habría que cuestionarse si realmente deberían aparecer artículo y adjetivo a un mismo nivel, ya que el adjetivo tiene función atributiva y puede ser omitido. Al lado derecho a un mismo nivel que Krähe, se halla el complemento mit Federn, y de ahí parten tanto el artículo determinado, el adjetivo como el atributo en genitivo der Pfauen, cuya única función es la de modificar mit Federn.

Este esquema corresponde en varios aspectos exactamente al análisis que hace la teoría de valencias Tesnière, en cuanto que sitúa el predicado en el centro del esquema como núcleo de la frase, del cual parten todos los complementos, sin diferenciar la función del sujeto de la de los objetos.

El hecho de que el sujeto esté situado en la parte izquierda del esquema, coincidiendo con el estema de Tesnière, se debe en nuestra opinión, más bien a fenómenos de orden sintáctico inherentes a las lenguas estudiadas, que suelen colocar en la 
cadena hablada el sujeto al comienzo de la frase. En efecto, esto no significa que en el alemán siempre sea así, pero es el orden sintáctico de una posición no marcada.

Pasamos al filólogo rumano Hariton Tiktin (1850-1936) quien utilizó en su Gramática romana $(1895)^{2}$ igualmente estemas y cuyo parecido predicado con los de Tesnière destacó E. Coseriu en el artículo Un précur seur méconnu de la syntaxe structurale: H. Tiktin, (1980). Para Tiktin la aplicación de un análisis lógico tiene la función de separar la frase en sus elementos para señalar así las relaciones existentes entre éllos. El estema sirve, pues, para tener una idea más clara de la estructura de la frase.

Según instrucciones de Tiktin, ha de averiguarse primero el predicado de la frase, después los demás elementos siguiendo un determinado orden. Finalmente se buscará para cada complemento el perteneciente atributo.

Los estemas utilizados por Tiktin, en efecto, se asemejan considerablemente a los de Tesnière, pues el predicado de la frase está en el centro en la parte superior del cual parten el sujeto y el objeto, ambos situados a un mismo nivel inferior. También aquí los adjetivos se hallan debajo de los sustantivos respectivos que modifican. Tiktin, Gramática romana, $\left(1895^{2}: 108\right)$.

$(3)^{10}$

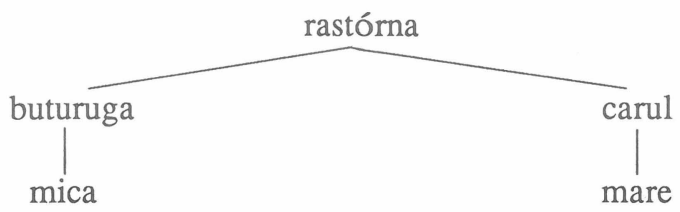

En cuanto a la representación de una frase, cuyo predicado es un verbo transitivo, encontramos analogías con los estemas de Tesnière. En lo único que sí se diferencian los estemas de Tiktín es cuando aparece a la vez un objeto en acusativo y otro en dativo, como es el caso en la siguiente frase: Tiktin, Gramática romana $\left(1895^{2}: 108\right)$.

$(4)^{11}$

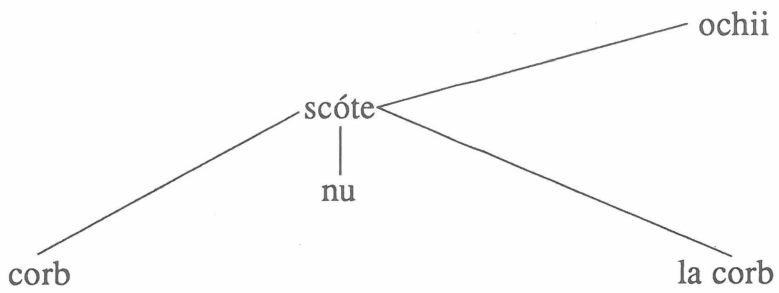

No hay razón convincente para situar al objeto de acusativo a un nivel incluso superior que el predicado de la frase, máxime cuando en (3) aparece el objeto en acusativo al lado derecho del predicado, al mismo nivel que el sujeto.

${ }^{10} \mathrm{La}$ traducción de la frase (3) es:

El leño pequeño tira el carro grande.

${ }^{11} \mathrm{La}$ traducciónde la frase (4) es:

Un cuervo no saca los ojos a otro cuervo. 
Cabe suponer que Tiktin no pensara en un orden jerárquico de las categorías sintácticas, teoría que formularía unos tres decenios después Otto Jespersen en Philosophy of Grammar (1924). Lo que implica el estema (4) es que el predicado se halla en el centro de la frase, pese a que carezca de una jerarquía sintáctica, y en ese sentido es perfectamente compatible con la concepción de un núcleo verbal de Lucien Tesnière.

Concluimos el trabajo con una representación de R. Blümel, Einführung in die Syntax, Heidelberg, 1914, como ejemplo de un enfoque científico que se caracteriza por su ineficacia explicativa, Heriner, Theorie der deuschen Syntax, (1973: 11,12).

Según Heringer (1973:11), Wackernagel (1926:64) calificó el formalismo de Blümel de «unanschaulich, formelhaft», y, en efecto, en comparación con los esquemas de Kern no tienen ninguna ventaja, ni tampoco tienen capacidad alguna de visualizar las estructuras sintácticas de una frase.

La construcción Karls jüngster Bruder es representada de la siguiente manera, según Heringer, Theorie, (1973:12).

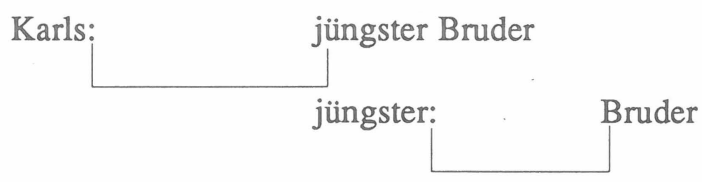

Según Heringer, Theorie (1973:12), Blümel utiliza el medio formal para representar la relación de dependencia. Debido a que no define el término «dependencia», las representaciones son poco claras. En efecto, el hecho de unir sintagmas por medio de líneas horizontales sin especificar la clase de relación entre éllos no contribuye a poner de manifiesto la estructura.

Además Blümel utiliza paréntesis para una representación lineal de relaciones sintácticas, pero sin denominar la función sintáctica de esos sintagmas.

$$
\text { Ich : [(habe... gesehen): dich]. }
$$

La representación pone de relieve, por medio del paréntesis, que el predicado un pretérito perfecto, consta de dos elementos, al ser un tiempo perifrástico. Los puntos suspensivos dentro del paréntesis corresponden a la posición sintáctica del alemán, ya que el verbo auxiliar va en segunda posición (después del sujeto) mientras que el participio perfecto va al final de la frase.

Por medio de los corchetes Blümel une lo que es el predicado y el objeto dich, y excluye el sujeto de la frase $i c h$. Todo parece indicar que Blümel asume una concepción de predicado tradicional, reflejada por la bisección de la frase, y en la que los objetos están más ligados al verbo, es decir, al núcleo de la frase, formando ambos el «predicado de la frase».

Teniendo en cuenta la fecha de aparición, el año 1914, el enfoque de Blümel es un intento de formalizar la descripción sintáctica en una gramática científica que actualmente tiene para nosotros tan solo un interés histórico, ya que Blümel no logra dar una idea clara de una estructura sintáctica al renunciar a la aplicación de las funciones 
sintácticas tradicionales. Pese a que Heringer, Theorie (1973:12) sostenga que haya utilizado el medio formal para representar la relación de dependencia, como ya indicábamos, consideramos que Blümel no ha logrado visualizar la estructura jerárquica de una frase, lo que reduce la capacidad de explicación de su formalismo considerablemente. Sólo nos cabe añadir que afortunadamente Blümel se propuso elaborar una sintaxis científica del alemán ya que de tener un objetivo pedagógico, los estudiantes difícilmente habrían podido servirse de sus representaciones.

Finalmente queremos hacer hincapie en que las representaciones gráficas son tan sólo un medio para visualizar la estructura de la frase. Obviamente no pueden separarse las representaciones gráficas de una concepción teórica. En élla la concepción de predicado desempeña un papel fundamental. Consideramos una concepción adecuada la que asume como centro estructural el núcleo verbal dentro de la frase, no sólo desde un punto de vista teórico sino también pedagógico, como ha demostrado la utilidad de los diccionarios de valencias bilingües y del alemán para la enseñanza sobre todo de una segunda lengua.

\section{Bibliografía}

Coseriu, Eugeniu, 1980. Un précurseur méconnu de la syntase structurale: H. Tiktin, en: Recherches de Linguistique, Hommage à Maurice Leroy, Tiré à part. Editions de lÙniversité de Bruxelles.

Eroms, Hans-Werner, 1981, Valenz Kasus und Präposition, Heidelberg Carl Winter Universitätsverlag.

Heringer, Hans Jürgen, 19732. Theorie der deutschen Syntax, Klaus Baumgärtner, Peter von Polenz, Hugo Steger (Ed.), München.

Kern, Franz, 1883. Die Deutsche Satzlehre. Eine Untersuchung ihrer Grundlagen. Berlín.

Tesnière, Lucien, 1953. Esquisse d'une Syntaxe Structurale, París Editions Klincksieck. Tesnière, Lucien, 19824 Eléments de Syntaxe Structurale, París Editions Klincksieck. Tiktin, Hariton, Gramatica romana pentru învetamîntul secundar. Teorie si practica, Bukarest, $1895^{2}$. 
